Bundesgesundheitsbl $2013 \cdot 56: 779-785$

DOI 10.1007/s00103-012-1651-8

Online publiziert: 27. Mai 2013

(c) Springer-Verlag Berlin Heidelberg 2013
G.B.M. Mensink - J. Truthmann - M. Rabenberg - C. Heidemann - M. Haftenberger · A. Schienkiewitz · A. Richter

Abteilung für Epidemiologie und Gesundheitsmonitoring, Robert Koch-Institut, Berlin

\section{Additional material online}

An English full-text version of this article is available at SpringerLink under supplementary material: dx.doi.org/10.1007/s00103-012-1651-8

\section{Hintergrund und Fragestellungen}

Die Zusammenstellung unserer Ernährung hat einen wesentlichen Einfluss auf unsere Gesundheit. Ein hoher Anteil Obst und Gemüse in der täglichen Kost ist dabei von Vorteil, da die pflanzlichen Lebensmittel wichtige Quellen für Vitamine, Mineralstoffe, Spurenelemente, Ballaststoffe und sekundäre Pflanzenstoffe darstellen. Sie weisen in der Regel einen hohen Wasseranteil und einen sehr geringen Fettgehalt auf und enthalten damit eine geringe Anzahl an Kalorien pro Volumeneinheit. Das günstige Verhältnis von hohem Nährstoff- und geringem Energiegehalt bei verhältnismäßig guter Sättigungswirkung macht die pflanzliche Nahrung aus ernährungsphysiologischer Sicht zu besonders wertvollen Lebensmitteln. Daneben kann ein hoher Anteil an Obst und Gemüse in der täglichen Ernährung dazu beitragen, geringere Mengen tierischer Lebensmittel und damit gleichzeitig weniger gesättigte Fettsäuren zu verzehren. Weiterführend weisen systematische Reviews auf die Bedeutung eines hohen Obst- und Gemüsekonsums hinsichtlich der Prävention verschiedener chronischer Krankheiten, z. B. koronarer Herzkrankheit, Hypertonie oder Schlaganfall, hin $[1,2,3]$.

Erwachsenen wird laut aktuellen Empfehlungen der Deutschen Gesellschaft für Ernährung (DGE) nahegelegt, täglich mindestens $400 \mathrm{~g}$ Gemüse und

\title{
Obst- und Gemüsekonsum in Deutschland
}

\section{Ergebnisse der Studie zur Gesundheit Erwachsener in Deutschland (DEGS1)}

250 g Obst zu konsumieren [4]. Parallel dazu gibt es in Deutschland seit dem Jahr 2002 die „5 am Tag“-Kampagne, die den Verzehr von 5 Portionen Obst und Gemüse pro Tag propagiert. Maximal eine Portion darf dabei durch ein Glas Obst- oder Gemüsesaft ersetzt werden. Dies entspricht für Erwachsene in etwa den empfohlenen Mengen der DGE [4]. In den vergangenen Jahren wurden diese Empfehlungen jedoch von einem Großteil der deutschen Bevölkerung nicht erreicht $[5,6,7]$. Mit der „Studie zur Gesundheit Erwachsener in Deutschland“ (DEGS) kann nun anhand repräsentativer Daten überprüft werden, wie hoch der aktuelle Obst- und Gemüsekonsum ist.

\section{Methoden}

DEGS ist Bestandteil des Gesundheitsmonitorings des Robert Koch-Instituts (RKI). Konzept und Design von DEGS sind an anderer Stelle ausführlich beschrieben $[8,9,10,11,12]$. Die erste Erhebungswelle (DEGS1) wurde von 2008 bis 2011 durchgeführt und umfasste Befragungen, Untersuchungen und Tests [13, 14]. Zielpopulation war die in Deutschland lebende Bevölkerung im Alter von 18 bis 79 Jahren. DEGS1 hat ein Mischdesign, das sowohl quer- als auch längsschnittliche Analysen ermöglicht. Hierbei wurde eine Einwohnermeldeamtsstichprobe gezogen, die ehemalige Teilnehmerinnen und Teilnehmer des Bun-
des-Gesundheitssurveys 1998 (BGS98) ergänzt. Insgesamt nahmen 8152 Personen teil, darunter 4193 Ersteingeladene (Response 42\%) und 3959 Teilnehmerinnen und Teilnehmer des BGS98 (Response 62\%). 7238 Personen besuchten eines der 180 Untersuchungszentren, 914 wurden ausschließlich befragt. Die Nettostichprobe $(n=7988)$ ermöglicht für den Altersbereich von 18 bis 79 Jahren repräsentative Querschnittsanalysen und Trendaussagen im Vergleich mit dem BGS98 (n=7124) [12]. Die Daten der erneut Teilnehmenden sind für Längsschnittanalysen nutzbar.

Zur Erfassung des Lebensmittelverzehrs erhielten die Teilnehmenden einige Tage vor dem Besuch im Untersuchungszentrum einen Ernährungsfragebogen, mit der Bitte, diesen ausgefüllt zum Untersuchungstermin mitzubringen. Der „semiquantitative Verzehrhäufigkeitsfragebogen“" erfasst die Verzehrhäufigkeiten und -mengen von insgesamt 53 Lebensmittelgruppen über einen Zeitraum von 4 Wochen und ist eine Weiterentwicklung des im Kinder- und Jugendgesundheitssurvey (KiGGS) eingesetzten Ernährungsfragebogens [5]. Die Modifikation betraf vor allem eine Anpassung der erfragten Lebensmittelportionen an die übliche Ernährungsweise von Erwachsenen. Hierzu wurden die Verzehrhäufigkeiten von Lebensmitteln aus dem Ernährungssurvey 1998 [15], einem Modul des BGS98, und der Nationalen Verzehrsstudie II (NVS II) [7] herangezo- 
gen. Sowohl der Ernährungsfragebogen von KiGGS [16] als auch der von DEGS1 [17] wurden validiert. Der DEGS1-Ernährungsfragebogen wurde bei $161 \mathrm{Er}$ wachsenen validiert, mit denen zusätzlich zum Ernährungsfragebogen jeweils 2-mal ein standardisiertes 24-StundenErnährungsinterview durchgeführt wurde. Die Einordnung der Verzehrmengen je Lebensmittelgruppe im gleichen oder anliegenden Quartil lag nach beiden Methoden zwischen $68 \%$ für gekochtes $\mathrm{Ge}$ müse und $94 \%$ für Kaffee, was einer zufriedenstellenden bis guten Validität entspricht [17].

Im DEGS1-Ernährungsfragebogen wird für die jeweilige Lebensmittelgruppe (bezogen auf die letzten 4 Wochen) die Frage gestellt: „Wie oft haben Sie ... gegessen?" (bzw. getrunken). Die vorliegende Auswertung bezieht sich auf folgende Lebensmittelgruppen: „Fruchtsaft (z. B. Orangen-, Apfel-, Kirschsaft)“, „Gemüsesaft (z. B. Tomaten-, Karottensaft)“, „frisches Obst (z. B. Apfel, Banane)“, ,gegartes Obst (z. B. Kompott, Konservenobst)“, „rohes Gemüse (z. B. Kopfsalat, Rohkost)“, „Hülsenfrüchte (z. B. Bohnen, Erbsen, Linsen) “ und "gegartes Gemüse".

Die Verzehrhäufigkeiten können mit den Kategorien „Nie“, „1 Mal im Monat“, „2-3 Mal im Monat", „1-2 Mal pro Woche“, „3-4 Mal pro Woche“, „5-6 Mal pro Woche“, „1 Mal am Tag“, „2 Mal am Tag“, „3 Mal am Tag“, ,4-5 Mal am Tag“ oder "Öfter als 5 mal am Tag" beantwortet werden. Bei der Portionsgröße kann z. B. zwischen „1/2 Portion (oder weniger) “, „1 Portion“, „2 Portionen“, „3 Portionen“ oder „4 Portionen (oder mehr)“ - je nach Lebensmittel auch „1/4 Portion" - gewählt werden. Standardportionen sind je nach Lebensmittelgruppe in verschiedenen Einheiten angegeben, z. B. als Glas, Tasse, Becher, Schüssel, Teller, Scheibe oder Stück. Zusätzlich sind zu einem Großteil der Fragen Fotos zur besseren Einschätzung der Portionsgrößen abgebildet. Aus den Häufigkeitsund Mengenangaben wurde der durchschnittliche Verzehr von Portionen pro Tag errechnet. Die Berechnungen erfolgten sowohl separat für die einzelnen Untergruppen (Obst, Gemüse, Säfte) als auch auf aggregierter Ebene. Ent- sprechend den Verzehrempfehlungen für Obst und Gemüse wurde auf aggregierter Ebene pro Tag bis zu eine Portion (ein Glas) konsumierter Obst- oder Gemüsesaft zum Obst- und Gemüsekonsum hinzugerechnet.

Zur Ermittlung der Veränderung des Obst- und Gemüseverzehrs in den letzten 10 Jahren wurden Daten des BGS98 herangezogen. Zusätzlich zu dem Ernährungssurvey 1998 wurden im allgemeinen Gesundheitsfragebogen des BGS98 auch Verzehrhäufigkeiten von verschiedenen Lebensmittelgruppen erfragt. Die zentrale Frage zur Verzehrhäufigkeit lautete: „Wie häufig nehmen Sie die einzelnen Nahrungsmittel bzw. Fertigprodukte zu sich? Bitte denken Sie an die letzten 12 Monate." Danach wurden die Lebensmittel tabellarisch aufgeführt, unter anderem „Frisch- oder Tiefkühlgemüse (gekocht)“, „Konservengemüse“, „Blattsalat, Rohkostsalat, rohes Gemüse (z. B. Tomaten, Möhren, Paprika)“, „, frisches Obst“. Eine weitere Frage lautete „Wie häufig nehmen Sie die einzelnen Getränke zu sich? Bitte denken Sie an die letzten 12 Monate.", wobei unter anderem „Obstund Gemüsesäfte" erfragt wurden. Die Antwortmöglichkeiten bei beiden Frageblöcken waren "mehrmals täglich“, „täglich bzw. fast täglich“, „mehrmals in der Woche“, „etwa 1 Mal in der Woche“, „2- bis 3 Mal im Monat“, „1 Mal im Monat oder seltener", und „(fast) nie“. Für die Auswertungen wurden die Antwortmöglichkeiten aus DEGS1 und BGS98 zum bestmöglichen Vergleich zusammengefasst. Zum Vergleich der Verzehrhäufigkeiten von Obst und Saft mit der BGS98-Antwortkategorie "mehrmals täglich“ wurden die DEGS1-Antwortkategorien „2 Mal am Tag" und höhere Häufigkeitsangaben kombiniert. Zum Vergleich der Verzehrhäufigkeiten von Gemüse wurde die Kategorie „fast täglich und öfter" gebildet. Hierfür wurden die BGS98-Antwortkategorien "mehrmals täglich“ und ,täglich bzw. fast täglich“, sowie die DEGS1-Antwortkategorien „5-6 Mal pro Woche“ und höhere Angaben zusammengefasst.

Der Sozialstatus wurde anhand eines Indexes bestimmt, in den Angaben zu schulischer und beruflicher Ausbildung, beruflicher Stellung sowie Haushaltsnet- toeinkommen (bedarfsgewichtet) eingehen und der eine Einteilung in niedrige, mittlere und hohe Statusgruppe ermöglicht [18].

Die Querschnittanalysen beziehen sich auf die 18- bis 79-Jährigen, die am Untersuchungsteil teilgenommen haben $(\mathrm{n}=7116)$, da nur diese Gruppe auch den DEGS-Ernährungsfragebogen ausgefüllt hat. Insgesamt wurden 7080 Ernährungsfragebögen ausgefüllt. Nach Plausibilitätsprüfungen wurden insgesamt 70 Teilnehmende ausgeschlossen, da deren Fragebögen sehr hohe (n=9) bzw. sehr geringe Verzehrmengen ( $n=53)$ aufwiesen oder unvollständig waren (mehr als 20 fehlende Werte; $n=8$ ). Somit wurden 7010 Teilnehmende in die Auswertung einbezogen. Die Trendanalysen beziehen sich auf die 18- bis 79-jährigen Teilnehmerinnen und Teilnehmer von BGS98 $(\mathrm{n}=7124)$ und DEGS1 $(\mathrm{n}=7010)$.

Die Querschnitts- und Trendanalysen werden mit einem Gewichtungsfaktor durchgeführt, der Abweichungen der Stichprobe von der Bevölkerungsstruktur (Stand 31.12.2010) hinsichtlich Alter, Geschlecht, Region und Staatsangehörigkeit sowie Gemeindetyp und Bildung korrigiert [12]. Für den Untersuchungsteil wurde ein gesonderter Gewichtungsfaktor erstellt. Bei der Berechnung der Gewichtung für die ehemaligen Teilnehmenden des BGS98 wurde die Wiederteilnahmewahrscheinlichkeit, basierend auf einem logistischen Regressionsmodell, berücksichtigt. Eine NonresponseAnalyse und der Vergleich einzelner erhobener Indikatoren mit Daten der amtlichen Statistik weisen auf eine hohe Repräsentativität der Stichprobe für die Wohnbevölkerung in Deutschland hin [12]. Um sowohl die Gewichtung als auch die Korrelation der Teilnehmenden innerhalb einer Gemeinde zu berücksichtigen, wurden die Konfidenzintervalle mit den Survey-Prozeduren für komplexe Stichproben von SAS 9.3 bestimmt. Unterschiede werden als statistisch signifikant angesehen, wenn sich die jeweiligen 95\%-Konfidenzintervalle nicht überschneiden. Auswertungen werden nach Geschlechts- und Altersgruppen (18 bis 29,30 bis 39,40 bis 49,50 bis 59,60 bis 69 und 70 bis 79 Jahre) sowie nach Sozialstatus dargestellt. 


\section{Ergebnisse}

In - Tab. 1 sind die Mittelwerte der individuell konsumierten Anzahl von Portionen pro Tag, für Obst und Gemüse insgesamt (inklusive bis zu einem Glas Saft) und separat für Obst, Gemüse und Obstund Gemüsesäfte nach Geschlecht und Alter dargestellt. Insgesamt konsumieren Frauen mit durchschnittlich 3,1 Portionen signifikant mehr Obst und Gemüse als Männer mit durchschnittlich 2,4 Portionen. Dies ist vor allem auf einen signifikant höheren Konsum an Obst sowie einen geringfügig, aber ebenfalls signifikant höheren Konsum an Gemüse zurückzuführen. Sowohl bei Frauen als auch bei Männern ist bis zur Altersgruppe 60 bis 69 Jahre eine Zunahme des Obstkonsums zu beobachten. Dagegen ist der Gemüseverzehr über die Altersgruppen fast konstant, während der Saftkonsum mit zunehmendem Alter leicht abnimmt.

In - Tab. 2 ist der Anteil an Personen, der bestimmte Portionsmengen an Obst und Gemüse insgesamt (inklusive bis zu einem Glas Saft) pro Tag erreicht, nach Geschlecht und Alter dargestellt. Dabei ist von besonderem Interesse der Anteil der Personen, der die „5 am Tag“-Empfehlung eingehalten hat. Dies erreichten mit $15,1 \%$ mehr als doppelt so viele Frauen wie Männer (7,0\%). Immerhin konsumieren 39,0\% der Frauen und 24,7\% der Männer mindestens 3 Portionen Obst und Gemüse am Tag. Im Altersvergleich zeigt sich die geringste Prävalenz bei Frauen, die mindestens 5 Portionen am Tag konsumieren, unter den 40 bis 49-Jährigen.

Der Anteil an Männern, der mindestens 5 Portionen konsumiert, nimmt im Altersgang bis zum Alter von 60 bis 69 Jahren zu. Bei beiden Geschlechtern ist der Anteil an Personen, der mindestens 3 Portionen pro Tag konsumiert, unter den 60- bis 69-Jährigen am höchsten. Etwa die Hälfte der Frauen und mehr als die Hälfte der Männer konsumieren eine bis unter 3 Portionen pro Tag. Der Anteil, der weniger als eine Portion pro Tag konsumiert, nimmt mit dem Alter ab.

In - Tab. 3 sind die Anteile der Personen, die bestimmte Portionsmengen

Bundesgesundheitsbl 2013 ·56:779-785 DOI 10.1007/s00103-012-1651-8

(c) Springer-Verlag Berlin Heidelberg 2013

G.B.M. Mensink · J. Truthmann - M. Rabenberg · C. Heidemann · M. Haftenberger · A. Schienkiewitz $\cdot$ A. Richter

Obst- und Gemüsekonsum in Deutschland. Ergebnisse der Studie zur Gesundheit Erwachsener in Deutschland (DEGS1)

\section{Zusammenfassung}

Bisher wurden die Empfehlungen der Deutschen Gesellschaft für Ernährung zum Obstund Gemüseverzehr von einem Großteil der Bevölkerung nicht erreicht. In der von 2008 bis 2011 durchgeführten ersten Welle der "Studie zur Gesundheit Erwachsener in Deutschland" (DEGS1) wurde in einer repräsentativen Stichprobe der 18- bis 79-jährigen Wohnbevölkerung $(n=7116)$ der Lebensmittelverzehr mit einem validierten Verzehrhäufigkeitsfragebogen ermittelt. Es wurde berechnet, wie viele Portionen Obst und Gemüse durchschnittlich pro Tag konsumiert werden und wie viele Personen die Empfehlungen von 5 Portionen Obst und Gemüse pro Tag erreichen. Im Mittel konsumieren Frauen 3,1 und Männer 2,4 Portionen Obst und Gemüse pro Tag. $15 \%$ der Frauen und $7 \%$ der
Männer erreichen die empfohlenen 5 Portionen pro Tag. Der Obstverzehr nimmt bei Frauen wie Männern bis zum Alter von 60 bis 69 Jahren zu. Mindestens 3 Portionen Obst und Gemüse pro Tag konsumieren 39\% der Frauen und 25\% der Männer. Der Anteil der Männer und Frauen, die täglich mindestens 3 Portionen verzehren, nimmt mit steigendem Sozialstatus tendenziell zu. Obwohl die Verzehrhäufigkeit von Obst gegenüber früheren Erhebungen leicht angestiegen ist, ist der Anteil der Personen, der 5 Portionen Obst und Gemüse am Tag konsumiert, immer noch sehr gering.

Schlüsselwörter

Gesundheitssurvey · Erwachsene - Obst . Gemüse - Verzehrhäufigkeit

\section{Fruit and vegetable intake in Germany. Results of the German Health Interview and Examination Survey for Adults (DEGS1)}

\section{Abstract}

To date, the recommendations of the German Nutrition Society on fruit and vegetable intake have not been met by the majority of the population. In the first wave of the "German Health Interview and Examination Survey for Adults" (DEGS1), which was conducted from 2008 to 2011, food consumption was determined with a validated food frequency questionnaire in a representative random sample of the 18-79-year-old resident population of Germany $(n=7,116)$. The number of portions of fruit and vegetables consumed on average every day and the number of persons meeting the recommended five portions of fruit and vegetables per day were calculated. On average, women consume 3.1 and men 2.4 portions of fruit and vegetables per day. Moreover, $15 \%$ of women and $7 \%$ of men reach the recommended quantity of five portions per day. Fruit intake increases in both men and women up to the age of 60 69 years. About $39 \%$ of women and $25 \%$ of men consume at least three portions of fruit and vegetables per day. The proportion of men and women who consume at least three portions per day tends to increase with rising socioeconomic status. Although the intake of fruit has increased slightly compared to previous surveys, the percentage of persons who consume five portions of fruit and vegetables per day is still very low. An English full-text version of this article is available at SpringerLink as supplemental.

Keywords Health survey · Adults · Fruit · Vegetables . Food frequency an Obst und Gemüse insgesamt (inklusive bis zu einem Glas Saft) pro Tag erreichen, nach Geschlecht und Sozialstatus dargestellt. Für beide Geschlechter nimmt mit steigendem Sozialstatus der Anteil an Personen, der 3 bis 5 bzw. 5 oder mehr Portionen pro Tag konsumiert, tendenziell (jedoch nicht signifikant) zu, während der Anteil, der weni- ger als eine Portion pro Tag konsumiert, signifikant abnimmt.

In - Tab. 4 sind sowohl die aus BGS98 als auch aus DEGS1 errechneten Anteile an Frauen und Männern dargestellt, die mehrmals pro Tag frisches Obst bzw. Fruchtsaft (BGS98: „Obst- und Gemüsesäfte") konsumieren. Weiterhin sind die errechneten Anteile an Frauen und Män- 
Tab. 1 Mittelwerte (95\%-Konfidenzintervalle) der durchschnittlich pro Tag konsumierten Portionen von Obst, Gemüse und Säften der erwachsenen deutschen Bevölkerung (DEGS1), nach Geschlecht und Altersgruppen ( $\mathrm{n}=7010)$

\begin{tabular}{|c|c|c|c|c|c|c|c|}
\hline $\begin{array}{l}\text { Alters- } \\
\text { gruppe }\end{array}$ & $\begin{array}{l}18 \text { bis } \\
29 \text { Jahre }\end{array}$ & $\begin{array}{l}30 \text { bis } \\
39 \text { Jahre }\end{array}$ & $\begin{array}{l}40 \text { bis } \\
49 \text { Jahre }\end{array}$ & $\begin{array}{l}50 \text { bis } \\
59 \text { Jahre }\end{array}$ & $\begin{array}{l}60 \text { bis } \\
69 \text { Jahre }\end{array}$ & $\begin{array}{l}70 \text { bis } \\
79 \text { Jahre }\end{array}$ & Gesamt \\
\hline \multicolumn{8}{|l|}{ Frauen } \\
\hline $\begin{array}{l}\text { Obst \& } \\
\text { Gemüse }^{\mathrm{a}}\end{array}$ & $\begin{array}{l}2,8 \\
(2,5-3,0)\end{array}$ & $\begin{array}{l}3,1 \\
(2,8-3,4)\end{array}$ & $\begin{array}{l}2,9 \\
(2,6-3,1)\end{array}$ & $\begin{array}{l}3,3 \\
(3,0-3,6)\end{array}$ & $\begin{array}{l}3,7 \\
(3,4-4,0)\end{array}$ & $\begin{array}{l}3,2 \\
(2,8-3,5)\end{array}$ & $\begin{array}{l}3,1 \\
(3,0-3,2)\end{array}$ \\
\hline Obst & $\begin{array}{l}1,4 \\
(1,2-1,6)\end{array}$ & $\begin{array}{l}1,6 \\
(1,3-1,8)\end{array}$ & $\begin{array}{l}1,5 \\
(1,3-1,6)\end{array}$ & $\begin{array}{l}1,9 \\
(1,7-2,2)\end{array}$ & $\begin{array}{l}2,3 \\
(2,0-2,6)\end{array}$ & $\begin{array}{l}2,0 \\
(1,7-2,3)\end{array}$ & $\begin{array}{l}1,8 \\
(1,6-1,9)\end{array}$ \\
\hline Gemüse & $\begin{array}{l}1,0 \\
(0,9-1,1)\end{array}$ & $\begin{array}{l}1,1 \\
(1,0-1,2)\end{array}$ & $\begin{array}{l}1,1 \\
(1,0-1,2)\end{array}$ & $\begin{array}{l}1,1 \\
(1,0-1,2)\end{array}$ & $\begin{array}{l}1,1 \\
(1,0-1,2)\end{array}$ & $\begin{array}{l}0,9 \\
(0,8-1,0)\end{array}$ & $\begin{array}{l}1,0 \\
(1,0-1,1)\end{array}$ \\
\hline Säfte & $\begin{array}{l}0,9 \\
(0,7-1,0)\end{array}$ & $\begin{array}{l}0,8 \\
(0,6-1,0)\end{array}$ & $\begin{array}{l}0,6 \\
(0,4-0,7)\end{array}$ & $\begin{array}{l}0,5 \\
(0,4-0,7)\end{array}$ & $\begin{array}{l}0,6 \\
(0,4-0,7)\end{array}$ & $\begin{array}{l}0,4 \\
(0,3-0,5)\end{array}$ & $\begin{array}{l}0,6 \\
(0,6-0,7)\end{array}$ \\
\hline \multicolumn{8}{|l|}{ Männer } \\
\hline $\begin{array}{l}\text { Obst \& } \\
\text { Gemüse }^{a}\end{array}$ & $\begin{array}{l}2,0 \\
(1,8-2,2)\end{array}$ & $\begin{array}{l}2,2 \\
(2,0-2,4)\end{array}$ & $\begin{array}{l}2,3 \\
(2,2-2,5)\end{array}$ & $\begin{array}{l}2,4 \\
(2,1-2,6)\end{array}$ & $\begin{array}{l}2,8 \\
(2,6-3,1)\end{array}$ & $\begin{array}{l}2,6 \\
(2,4-2,8)\end{array}$ & $\begin{array}{l}2,4 \\
(2,3-2,4)\end{array}$ \\
\hline Obst & $\begin{array}{l}0,8 \\
(0,7-0,9)\end{array}$ & $\begin{array}{l}1,0 \\
(0,9-1,2)\end{array}$ & $\begin{array}{l}1,2 \\
(1,1-1,3)\end{array}$ & $\begin{array}{l}1,3 \\
(1,2-1,5)\end{array}$ & $\begin{array}{l}1,7 \\
(1,5-1,9)\end{array}$ & $\begin{array}{l}1,5 \\
(1,4-1,7)\end{array}$ & $\begin{array}{l}1,2 \\
(1,2-1,3)\end{array}$ \\
\hline Gemüse & $\begin{array}{l}0,7 \\
(0,6-0,8)\end{array}$ & $\begin{array}{l}0,7 \\
(0,7-0,8)\end{array}$ & $\begin{array}{l}0,8 \\
(0,7-0,8)\end{array}$ & $\begin{array}{l}0,7 \\
(0,7-0,8)\end{array}$ & $\begin{array}{l}0,8 \\
(0,7-0,9)\end{array}$ & $\begin{array}{l}0,8 \\
(0,7-0,8)\end{array}$ & $\begin{array}{l}0,8 \\
(0,7-0,8)\end{array}$ \\
\hline Säfte & $\begin{array}{l}1,1 \\
(0,9-1,2)\end{array}$ & $\begin{array}{l}0,9 \\
(0,7-1,2)\end{array}$ & $\begin{array}{l}0,7 \\
(0,5-0,8)\end{array}$ & $\begin{array}{l}0,7 \\
(0,5-0,8)\end{array}$ & $\begin{array}{l}0,5 \\
(0,4-0,7)\end{array}$ & $\begin{array}{l}0,5 \\
(0,4-0,7)\end{array}$ & $\begin{array}{l}0,7 \\
(0,7-0,8)\end{array}$ \\
\hline
\end{tabular}

nern, die fast täglich oder häufiger rohes Gemüse (BGS98: „Blattsalat, Rohkostsalat, rohes Gemüse") bzw. gegartes Gemüse [BGS98: „Frisch- oder Tiefkühlgemüse (gekocht)"] konsumieren, dargestellt. Der Anteil der Frauen, der mehrmals täglich Obst isst, hat von $18,5 \%$ im BGS98 gegenüber $26,2 \%$ in DEGS1 deutlich zugenommen. Der Anteil an Männern, der mehrmals am Tag Obst isst, ist deutlich geringer als bei Frauen. Auch hier ist der Anteil in DEGS1 mit 13,9\% höher als im BGS98 mit 9,8\%. Während für den Anteil an Personen, der mehrmals am Tag Obst isst, in DEGS1 ein Anstieg mit zunehmendem Alter zu sehen ist, ist dies im BGS98 nicht der Fall. Der Anteil an Personen, der mehrmals am Tag Saft zu sich nimmt, hat seit dem BGS98 bei Frauen und noch stärker bei Männern zugenommen. Insgesamt ist in DEGS1 der Anteil bei Frauen und Männern etwa gleich. Der Anteil derjenigen, der mehrmals am Tag Saft konsumiert, nimmt im BGS98 mit steigendem Alter ab, in DEGS1 zeigt sich hierfür kein klarer Altersunterschied. Der Anteil an Personen, der fast täglich oder mehrmals am Tag rohes Gemüse zu sich nimmt, ist bei Frauen höher als bei Männern. Zwischen BGS98 und DEGS1 zeigt sich sowohl bei Frauen als auch bei Männern ein deut- licher und signifikanter Rückgang. Eine deutliche $\mathrm{Zu}$ - oder Abnahme mit dem Alter ist in beiden Surveys nicht erkennbar. Der Anteil derjenigen, der fast täglich oder mehrmals am Tag gegartes Gemüse zu sich nimmt, ist bei Frauen höher als bei Männern. Zwischen BGS98 und DEGS1 hat dieser Anteil bei Frauen leicht und bei Männern deutlich abgenommen, dies ist für beide Geschlechter insgesamt und in einigen Altersgruppen signifikant. Dieser Rückgang gilt für alle Altersgruppen außer für 18- bis 29-jährige Frauen.

\section{Diskussion}

Im Mittel konsumieren Frauen 3,1 und Männer 2,4 Portionen Obst und Gemüse pro Tag, die empfohlenen 5 Portionen pro Tag erreichen 15\% der Frauen und 7\% der Männer. Immerhin konsumieren 39\% der Frauen und 25\% der Männer mindestens 3 Portionen Obst und Gemüse pro Tag. In allen Altersgruppen konsumieren Frauen deutlich häufiger Obst und Gemüse als Männer. Dieses Ergebnis passt zu früheren Studien, die darauf hindeuten, dass Frauen sich gesundheitsbewusster ernähren als Männer $[19,20]$. Interessant ist die Beobachtung, dass der Obst- und Gemüsekon- sum mit zunehmendem Alter, bis etwa 60 bis 69 Jahre, steigt, obwohl der Energiebedarf mit dem Alter abnimmt. Dies deutet auf ein steigendes Gesundheitsbewusstsein im Alter hin. Der Anstieg des Obst- und Gemüsekonsums scheint in den letzten Jahren besonders in den oberen Altersgruppen stattgefunden zu haben. Der Konsum von Obst- und Gemüsesäften ist dagegen in den jüngeren Altersgruppen am höchsten.

Die Verzehrhäufigkeiten aus DEGS1 wurden den Angaben aus dem BGS98 auf einer sehr groben Ebene gegenübergestellt. Ein detaillierterer Vergleich beider Verzehrangaben ist durch methodische Unterschiede nicht möglich. Im BGS98 waren Fragen zur Verzehrhäufigkeit in den allgemeinen Gesundheitsfragebogen integriert, während in DEGS1 ein separater Ernährungsfragebogen eingesetzt wurde. Der Referenzzeitraum bezog sich im BGS98 auf die letzten $12 \mathrm{Mo}$ nate und in DEGS1 auf die letzten 4 Wochen. Im BGS98 wurden keine Portionsmengen erfragt [21]. Außerdem unterschieden sich die Antwortkategorien zur Erfassung der Verzehrhäufigkeit zwischen beiden Surveys leicht, und die Lebensmittelgruppen wurden teilweise unterschiedlich definiert. Dies betraf unter anderem Säfte und Gemüse. Im BGS98 wurden „Blattsalat, Rohkostsalat, rohes Gemüse“ sowie „Obst- und Gemüsesäfte " jeweils in einer Frage erfasst. Die erste Gruppierung lässt sich nach unserer Einschätzung gut mit der Frage zu rohem Gemüse aus DEGS1 vergleichen. In DEGS1 wurden jedoch getrennte Fragen zum Verzehr von Obst- und von Gemüsesaft gestellt. Die Verzehrhäufigkeit von Gemüsesaft kann wegen der fehlenden Portionsangaben im BGS98 nicht einfach zu der von Obstsaft addiert werden, da eine Kombination von z. B. „mehrmals in der Woche" für beide Lebensmittelgruppen bedeuten kann, dass täglich Säfte getrunken werden, aber nicht zwangsläufig. Die Konsumhäufigkeit von Gemüsesäften war in DEGS1 jedoch sehr gering und ist für den Vergleich der Lebensmittelgruppen somit vernachlässigbar. Auch die Fragen zum Verzehr von gegartem Gemüse unterschieden sich: Im BGS98 wurden gekochtes Frischoder Tiefkühlgemüse (eine Frage) so- 
wie Konservengemüse getrennt erfasst, während in DEGS1 gegartes Gemüse ohne weitere Differenzierung und Hülsenfrüchte getrennt erfasst wurden. Sowohl für Hülsenfrüchte in DEGS1 als auch Konservengemüse in BGS98 waren die Angaben sehr niedrig, sodass dieser Unterschied nicht ins Gewicht fällt. Dagegen wurde frisches Obst in beiden Surveys etwa gleich erfasst.

Unter Vorbehalt der oben genannten methodischen Differenzen zeigt sich, dass der Anteil der Männer und Frauen, der mehrmals täglich Obst verzehrt, zugenommen hat. Diese Beobachtung wird durch die steigenden Verbrauchszahlen für Obst aus der Agrarstatistik bestätigt [22]. Die Zunahme zwischen BGS98 und DEGS1 ist jedoch nur bei Männern und Frauen in den Altersgruppen 60 bis 69 und 70 bis 79 Jahren signifikant und bei Frauen im Alter von 30 bis 39 Jahren. Möglicherweise sind gerade Personen dieser Altersgruppen empfänglich für präventive Maßnahmen. Eine weitere positive Entwicklung betrifft die Zunahme des Anteils der Männer und Frauen, der mehrmals täglich Saft konsumiert. Hingegen hat sich der Anteil der Männer und Frauen, der fast täglich rohes Gemüse verzehrt, und der Anteil der Männer, der täglich gegartes Gemüse verzehrt, leicht verschlechtert. Einen Einfluss der EHEC-Krise im Jahr 2011 [23] wäre denkbar, jedoch zeigten erste Analysen dazu keinen starken Rückgang des Konsums in den entsprechenden Monaten.

Es gab in den letzten Jahren weitere bundesweit repräsentative Erhebungen zum Obst- und Gemüsekonsum. In der Studie "Gesundheit in Deutschland aktuell 2010 (GEDA 2010)“, die Teil des Gesundheitsmonitorings am Robert KochInstitut ist, wurden rund 22.000 Personen telefonisch befragt [24]. Der Befragungszeitraum (September 2009 bis Juli 2010) liegt innerhalb des DEGS1-Erhebungszeitraums. Der Anteil an Personen, der 5 Portionen Obst- und Gemüse pro Tag verzehrte, ist in GEDA 2010 etwas geringer als in DEGS1 (Frauen 12\% vs. $15 \%$, Männer $5 \%$ vs. $7 \%$ ). Der Unterschied ist vermutlich auf die Zugangswege und die Abfrage zurückzuführen. In der schriftlichen Erfassung in DEGS1

Tab. 2 Anteil an Personen (Prozent und 95\%-Konfidenzintervalle) in Kategorien der durchschnittlich pro Tag konsumierten Portionen von Obst, Gemüse und Säften ${ }^{\mathrm{a}}$ der erwachsenen deutschen Bevölkerung (DEGS1), nach Geschlecht und Altersgruppen ( $\mathrm{n}=7010)$

\begin{tabular}{|c|c|c|c|c|c|c|c|}
\hline $\begin{array}{l}\text { Alters- } \\
\text { gruppe }\end{array}$ & $\begin{array}{l}18 \text { bis } \\
29 \text { Jahre }\end{array}$ & $\begin{array}{l}30 \text { bis } \\
39 \text { Jahre }\end{array}$ & $\begin{array}{l}40 \text { bis } \\
49 \text { Jahre }\end{array}$ & $\begin{array}{l}50 \text { bis } \\
59 \text { Jahre }\end{array}$ & $\begin{array}{l}60 \text { bis } \\
69 \text { Jahre }\end{array}$ & $\begin{array}{l}70 \text { bis } \\
79 \text { Jahre }\end{array}$ & Gesamt \\
\hline \multicolumn{8}{|l|}{ Frauen } \\
\hline $\begin{array}{l}\text { Weniger } \\
\text { als eine } \\
\text { Portion }\end{array}$ & $\begin{array}{l}18,5 \\
(14,8- \\
22,8)\end{array}$ & $\begin{array}{l}10,9 \\
(8,0- \\
14,8)\end{array}$ & $\begin{array}{l}13,4 \\
(10,3- \\
17,2)\end{array}$ & $\begin{array}{l}12,1 \\
(9,0- \\
15,9)\end{array}$ & $\begin{array}{l}6,0 \\
(4,4- \\
8,2)\end{array}$ & $\begin{array}{l}7,7 \\
(5,3- \\
11,2)\end{array}$ & $\begin{array}{l}11,9 \\
(10,5- \\
13,4)\end{array}$ \\
\hline $\begin{array}{l}\text { Eine bis } \\
\text { unter } \\
3 \text { Por- } \\
\text { tionen }\end{array}$ & $\begin{array}{l}48,3 \\
(43,2- \\
53,4)\end{array}$ & $\begin{array}{l}52,9 \\
(46,7- \\
58,9)\end{array}$ & $\begin{array}{l}52,4 \\
(47,8- \\
56,9)\end{array}$ & $\begin{array}{l}45,5 \\
(40,7- \\
50,4)\end{array}$ & $\begin{array}{l}44,9 \\
(40,2- \\
49,6)\end{array}$ & $\begin{array}{l}51,2 \\
(45,7- \\
56,7)\end{array}$ & $\begin{array}{l}49,2 \\
(47,0- \\
51,4)\end{array}$ \\
\hline $\begin{array}{l}\text { Drei bis } \\
\text { unter } \\
5 \text { Por- } \\
\text { tionen }\end{array}$ & $\begin{array}{l}19,5 \\
(15,8- \\
23,7)\end{array}$ & $\begin{array}{l}21,4 \\
(16,7- \\
26,9)\end{array}$ & $\begin{array}{l}23,2 \\
(19,8- \\
27,1)\end{array}$ & $\begin{array}{l}24,6 \\
(21,3- \\
28,4)\end{array}$ & $\begin{array}{l}30,5 \\
(25,8- \\
35,5)\end{array}$ & $\begin{array}{l}25,3 \\
(20,8- \\
30,3)\end{array}$ & $\begin{array}{l}23,9 \\
(22,2- \\
25,6)\end{array}$ \\
\hline $\begin{array}{l}\text { Fünf } \\
\text { Portionen } \\
\text { oder } \\
\text { mehr }\end{array}$ & $\begin{array}{l}13,8 \\
(10,6- \\
17,8)\end{array}$ & $\begin{array}{l}14,8 \\
(10,9- \\
19,8)\end{array}$ & $\begin{array}{l}11,0 \\
(8,8- \\
13,7)\end{array}$ & $\begin{array}{l}17,8 \\
(14,2- \\
22,2)\end{array}$ & $\begin{array}{l}18,6 \\
(15,2- \\
22,7)\end{array}$ & $\begin{array}{l}15,8 \\
(11,9- \\
20,7)\end{array}$ & $\begin{array}{l}15,1 \\
(13,6- \\
16,7)\end{array}$ \\
\hline \multicolumn{8}{|l|}{ Männer } \\
\hline $\begin{array}{l}\text { Weniger } \\
\text { als eine } \\
\text { Portion }\end{array}$ & $\begin{array}{l}25,3 \\
(21,1- \\
30,0)\end{array}$ & $\begin{array}{l}23,2 \\
(18,6- \\
28,5)\end{array}$ & $\begin{array}{l}16,8 \\
(13,5- \\
20,7)\end{array}$ & $\begin{array}{l}21,6 \\
(17,4- \\
26,3)\end{array}$ & $\begin{array}{l}12,4(9,6- \\
15,9)\end{array}$ & $\begin{array}{l}13,1(9,9- \\
17,3)\end{array}$ & $\begin{array}{l}19,3 \\
(17,7- \\
21,0)\end{array}$ \\
\hline $\begin{array}{l}\text { Eine bis } \\
\text { unter } \\
3 \text { Por- } \\
\text { tionen }\end{array}$ & $\begin{array}{l}55,6 \\
(51,0- \\
60,1)\end{array}$ & $\begin{array}{l}54,9 \\
(48,5- \\
61,1)\end{array}$ & $\begin{array}{l}60,5 \\
(55,7- \\
65,1)\end{array}$ & $\begin{array}{l}52,4 \\
(47,5- \\
57,3)\end{array}$ & $\begin{array}{l}55,2 \\
(49,9- \\
60,3)\end{array}$ & $\begin{array}{l}56,5 \\
(51,0- \\
61,7)\end{array}$ & $\begin{array}{l}56,0 \\
(53,7- \\
58,3)\end{array}$ \\
\hline $\begin{array}{l}\text { Drei bis } \\
\text { unter } \\
5 \text { Por- } \\
\text { tionen }\end{array}$ & $\begin{array}{l}14,1 \\
(10,9- \\
18,1)\end{array}$ & $\begin{array}{l}16,3 \\
(12,2- \\
21,3)\end{array}$ & $\begin{array}{l}16,8 \\
(13,8- \\
20,3)\end{array}$ & $\begin{array}{l}19,3 \\
(15,8- \\
23,5)\end{array}$ & $\begin{array}{l}20,8 \\
(16,6- \\
25,7)\end{array}$ & $\begin{array}{l}20,8 \\
(16,8- \\
25,6)\end{array}$ & $\begin{array}{l}17,7 \\
(16,1- \\
19,4)\end{array}$ \\
\hline $\begin{array}{l}\text { Fünf } \\
\text { Portionen } \\
\text { oder } \\
\text { mehr }\end{array}$ & $\begin{array}{l}5,0 \\
(3,1- \\
8,0)\end{array}$ & $\begin{array}{l}5,7 \\
(3,4- \\
9,4)\end{array}$ & $\begin{array}{l}5,9 \\
(4,1- \\
8,5)\end{array}$ & $\begin{array}{l}6,7 \\
(4,8- \\
9,3)\end{array}$ & $\begin{array}{l}11,6 \\
(8,5- \\
15,7)\end{array}$ & $\begin{array}{l}9,5 \\
(7,0- \\
12,8)\end{array}$ & $\begin{array}{l}7,0 \\
(6,1- \\
8,1)\end{array}$ \\
\hline
\end{tabular}

Obst, Gemüse und bis zu ein Glas Obst- oder Gemüsesaft.

Tab. 3 Anteil an Personen (Prozent und 95\%-Konfidenzintervalle) in Kategorien der durchschnittlich pro Tag konsumierten Portionen von Obst, Gemüse und Säften ${ }^{\mathrm{a}}$ der erwachsenen deutschen Bevölkerung (DEGS1), nach Geschlecht und Sozialstatusgruppen ( $n=7010)$

\begin{tabular}{|lllll}
$\begin{array}{l}\text { Obst, Gemüse } \\
\text { und Saft pro Tag }\end{array}$ & $\begin{array}{l}\text { Weniger als eine } \\
\text { Portion }\end{array}$ & $\begin{array}{l}\text { Eine bis unter } \\
\text { 3 Portionen }\end{array}$ & $\begin{array}{l}\text { Drei bis unter } \\
\text { 5 Portionen }\end{array}$ & $\begin{array}{l}\text { Fünf Portionen } \\
\text { oder mehr }\end{array}$ \\
\hline Frauen & & & & \\
\hline Niedrig & $17,5(14,3-21,3)$ & $49,3(44,2-54,4)$ & $19,5(15,8-23,8)$ & $13,6(10,7-17,2)$ \\
\hline Mittel & $11,6(9,8-13,6)$ & $50,8(48,0-53,6)$ & $23,2(21,2-25,4)$ & $14,3(12,6-16,3)$ \\
\hline Hoch & $6,6(4,8-9,0)$ & $43,6(39,1-48,1)$ & $31,6(27,3-36,2)$ & $18,2(15,0-21,9)$ \\
\hline Männer & & & & \\
\hline Niedrig & $29,2(24,5-34,4)$ & $50,3(44,4-56,2)$ & $14,9(11,5-19,3)$ & $5,6(3,7-8,3)$ \\
\hline Mittel & $18,3(16,2-20,5)$ & $57,8(54,8-60,8)$ & $17,1(15,1-19,3)$ & $6,8(5,6-8,3)$ \\
\hline Hoch & $14,0(11,4-17,2)$ & $55,8(51,3-60,2)$ & $21,2(18,0-24,9)$ & $8,9(6,9-11,5)$ \\
\hline${ }^{a}$ Obst, Gemüse und bis zu ein Glas Obst- oder Gemüsesaft. & & \\
\hline
\end{tabular}

war eine etwas detailliertere Abfrage der Lebensmittel möglich, während die telefonische Befragung in GEDA lediglich 3 einfache Fragen zum Obst- und Gemüse- verzehr umfasste. Mit zunehmendem Alter wurde jedoch in beiden Surveys eine Steigerung der konsumierten Portionen Obst und Gemüse pro Tag beobach- 
Tab. 4 Anteil an Personen (Prozent und 95\%-Konfidenzintervalle) in Kategorien der durchschnittlichen Konsumhäufigkeit von frischem Obst, (Frucht-)Säften, rohem Gemüse und gegartem Gemüse für BGS98 $(n=7124)$ und DEGS1 ( $n=7010)$, nach Altersgruppen

\begin{tabular}{|c|c|c|c|c|c|c|c|}
\hline $\begin{array}{l}\text { Alters- } \\
\text { gruppe }\end{array}$ & 18 bis 29 Jahre & 30 bis 39 Jahre & 40 bis 49 Jahre & 50 bis 59 Jahre & 60 bis 69 Jahre & 70 bis 79 Jahre & Gesamt \\
\hline \multicolumn{8}{|c|}{ Frisches Obst mehrmals/Tag } \\
\hline \multicolumn{8}{|l|}{ Frauen } \\
\hline BGS98 & $17,3(14,1-21,1)$ & $15,8(12,9-19,3)$ & $19,3(16,2-22,8)$ & $23,4(19,6-27,6)$ & $16,9(12,8-22,1)$ & $16,6(12,1-22,2)$ & $18,5(16,7-20,3)$ \\
\hline DEGS1 & $20,5(15,8-26,2)$ & $24,1(19,5-29,5)$ & $22,1(18,6-26,0)$ & $27,5(24,1-31,2)$ & $33,4(28,7-38,4)$ & $32,9(27,4-38,9)$ & $26,2(24,2-28,3)$ \\
\hline \multicolumn{8}{|l|}{ Männer } \\
\hline BGS98 & $7,9(5,5-11,1)$ & $8,3(6,3-10,8)$ & $11,7(8,8-15,5)$ & $11,1(8,6-14,2)$ & $9,0(6,2-12,8)$ & $9,8(6,0-15,6)$ & $9,8(8,5-11,2)$ \\
\hline DEGS1 & $6,4(4,3-9,4)$ & $11,2(7,8-15,8)$ & $13,5(10,6-17,1)$ & $15,7(12,7-19,3)$ & $20,5(16,5-25,2)$ & $20,1(16,6-24,2)$ & $13,9(12,6-15,4)$ \\
\hline \multicolumn{8}{|c|}{ Säfte mehrmals/Tag } \\
\hline \multicolumn{8}{|l|}{ Frauen } \\
\hline BGS98 & $14,6(11,6-18,4)$ & $8,4(6,6-10,7)$ & $7,9(6,0-10,4)$ & $6,5(4,8-8,6)$ & $4,7(3,2-7,0)$ & $4,2(2,3-7,4)$ & $7,9(7,0-9,0)$ \\
\hline DEGS1 & $12,9(10,3-16,1)$ & $21,9(17,0-27,8)$ & $12,9(10,2-16,2)$ & $10,5(8,1-13,6)$ & $12,8(9,7-16,7)$ & $9,0(6,4-12,6)$ & $13,2(11,7-14,9)$ \\
\hline \multicolumn{8}{|l|}{ Männer } \\
\hline BGS98 & $6,8(5,0-9,2)$ & $5,4(3,8-7,6)$ & $4,2(2,8-6,3)$ & $4,2(2,7-6,6)$ & $3,7(2,2-6,1)$ & $2,8(1,3-5,6)$ & $4,6(3,9-5,5)$ \\
\hline DEGS1 & $14,7(11,7-18,4)$ & $16,2(12,1-21,2)$ & $13,4(10,4-17,2)$ & $10,9(8,2-14,3)$ & $10,9(7,9-14,7)$ & $13,2(9,3-18,5)$ & $13,2(11,8-14,9)$ \\
\hline \multicolumn{8}{|c|}{ Rohes Gemüse fast täglich und öfter } \\
\hline \multicolumn{8}{|l|}{ Frauen } \\
\hline BGS98 & $36,5(32,8-40,4)$ & $38,2(33,6-43,0)$ & $45,6(41,3-50,1)$ & $47,7(42,9-52,6)$ & $39,7(34,0-45,8)$ & $35,4(28,6-42,9)$ & $41,0(38,7-43,4)$ \\
\hline DEGS1 & $28,7(24,3-33,4)$ & $25,5(20,7-30,9)$ & $29,1(25,0-33,6)$ & $29,5(25,5-33,8)$ & $29,3(24,7-34,3)$ & $23,6(19,5-28,4)$ & $27,8(25,8-30,0)$ \\
\hline \multicolumn{8}{|l|}{ Männer } \\
\hline BGS98 & $25,6(21,8-29,9)$ & $25,4(21,6-29,5)$ & $28,1(24,0-32,5)$ & $32,7(28,6-37,1)$ & $35,2(31,0-39,7)$ & $28,8(21,7-37,1)$ & $29,1(26,9-31,4)$ \\
\hline DEGS1 & $15,2(11,9-19,2)$ & $17,9(13,4-23,6)$ & $18,4(14,2-23,6)$ & $17,1(13,7-21,1)$ & $20,0(15,8-24,9)$ & $18,7(14,8-23,3)$ & $17,7(15,7-19,9)$ \\
\hline \multicolumn{8}{|c|}{ Gegartes Gemüse fast täglich und öfter } \\
\hline \multicolumn{8}{|l|}{ Frauen } \\
\hline BGS98 & $12,2(9,3-15,8)$ & $16,0(12,9-19,6)$ & $19,7(16,3-23,7)$ & $20,1(16,7-24,0)$ & $25,2(20,6-30,4)$ & $27,8(21,6-35,0)$ & $19,8(17,8-21,9)$ \\
\hline DEGS1 & $15,0(11,5-19,4)$ & $14,4(10,6-19,3)$ & $15,0(11,8-19,1)$ & $16,3(13,2-19,9)$ & $18,2(14,5-22,6)$ & $17,3(13,2-22,4)$ & $15,9(14,3-17,7)$ \\
\hline \multicolumn{8}{|l|}{ Männer } \\
\hline BGS98 & $12,1(9,4-15,6)$ & $13,0(10,3-16,3)$ & $11,6(9,1-14,8)$ & $13,6(10,6-17,2)$ & $18,6(14,2-24,0)$ & $16,8(12,0-23,0)$ & $13,9(11,9-16,0)$ \\
\hline DEGS1 & $7,2(4,9-10,4)$ & $7,4(4,8-11,0)$ & $6,6(4,4-9,8)$ & $6,1(4,3-8,7)$ & $8,8(5,7-13,4)$ & $8,6(6,4-11,5)$ & $7,3(6,2-8,5)$ \\
\hline
\end{tabular}

tet. Während die Prävalenzunterschiede bei Frauen im Altersgang geringfügiger ausfielen, war die Differenz bei Männer ab dem 60. Lebensjahr besonders deutlich (DEGS1: 60 bis 69 Jahre 12\%, 70 bis 79 Jahre 10\%; GEDA 2010: 60 bis 69 Jahre $5 \%, 70$ bis 79 Jahre $4 \%$ ).

In der NVS II, wurden von 2005 bis 2006 deutschlandweit repräsentative Verzehrdaten unter anderem für Obst und Gemüse sowie Säfte erfasst [7]. Auch zwischen NVS II und DEGS1 gibt es Einschränkungen im Hinblick auf die Vergleichbarkeit, da die NVS II quantitativere Erhebungsinstrumente eingesetzt hat und ihre Ergebnisse entsprechend als Grammmengen präsentieren konnte. Nichtsdestotrotz wurde auch in der NVS II ein steigender Obstkonsum mit zunehmendem Alter beobachtet, wobei die höchsten Verzehrmengen bei den 65- bis 80-jährigen Männern und den 51- bis 64-jährigen Frauen beobachtet wurden [7]. Ebenso wurde in der NVS II ein höherer Konsum an Obst und Gemüse bei höherem Sozialstatus festgestellt. Während in DEGS1 kaum ein Unterschied bei der Anzahl einzelner Gemüseportionen pro Tag in den Altersgruppen zu sehen war, nahmen laut NVS II die Verzehrmengen für Gemüse mit steigendem Alter zu. Dies ist vermutlich auf die deutlich detailliertere Erfassung der einzelnen Gemüsearten zurückzuführen.

Bei allen hier herangezogenen Erhebungen zum Obst- und Gemüsekonsum gibt es methodische Unterschiede in der Auswahl der Erhebungsinstrumente, bei der Stichprobenziehung und in der Wahl der Zugangswege (telefonische, schriftliche und „Face-to-face-Befragung“). Dies erschwert die Vergleichbarkeit der ein- zelnen Studien. Außerdem gibt es Unterschiede im Referenzzeitraum, wodurch die Erinnerungsfähigkeit der Teilnehmerinnen und Teilnehmer unterschiedlich beansprucht wird und auch saisonale Schwankungen unterschiedlich erfasst werden.

\section{Fazit}

Der Obstverzehr ist gegenüber früheren Erhebungen leicht angestiegen. Der Anteil an Personen, der die Empfehlungen von 5 Portionen Obst und Gemüse pro Tag erreicht, ist immer noch sehr gering. 


\section{Korrespondenzadresse}

\section{G.B.M. Mensink}

Abteilung für Epidemiologie und

Gesundheitsmonitoring,

Robert Koch-Institut

General-Pape-Str. 62-66, 12101 Berlin

g.mensink@rki.de

Finanzierung der Studie. Die Studie wurde finanziert mit Mitteln des Robert Koch-Instituts und des Bundesministeriums für Gesundheit.

Interessenkonflikt. Der korrespondierende Autor gibt für sich und seine Koautoren an, dass kein Interessenkonflikt besteht.

\section{Literatur}

1. Boeing H, Bechthold A, Bub A et al (2012) Critical review: vegetables and fruit in the prevention of chronic diseases. Eur J Nutr 51:637-663

2. World Cancer Research Fund/American Institute for Cancer Research (2007) Food, nutrition, physical activity, and the prevention of cancer: a global perspective. AICR, Washington DC

3. World Health Organization (2003) Diet, nutrition and the prevention of chronic diseases. Report of a joint WHO/FAO expert consultation. WHO, Geneva

4. Deutsche Gesellschaft für Ernährung (2012) Jetzt mit 5 am Tag in die Saison starten. In: DGE aktuell - Presseinformation. Deutsche Gesellschaft für Ernährung e.V., Bonn, http://www.dge.de

5. Mensink GBM, Burger M (2004) Was isst du? Ein Verzehrshäufigkeitsfragebogen für Kinder und Jugendliche. Bundesgesundheitsbl Gesundheitsforsch Gesundheitsschutz 47:219-226

6. Rabenberg M, Mensink GBM (2011) Obst- und Gemüsekonsum heute. In: GBE kompakt 2 (6). Robert Koch-Institut, Berlin

7. Max Rubner Institut (2008) Nationale Verzehrsstudie II - Ergebnisbericht, Teil 2. Max Rubner Institut - Bundesforschungsinstitut für Ernährung und Lebensmittel, Karlsruhe

8. Kurth BM, Lange C, Kamtsiuris P, Hölling H (2009) Gesundheitsmonitoring am Robert Koch-Institut. Sachstand und Perspektiven. Bundesgesundheitsbl Gesundheitsforsch Gesundheitsschutz 52:557-570

9. Kurth BM (2012) Das RKI-Gesundheitsmonitoring - was es enthält und wie es genutzt werden kann. Public Health Forum 20(76):4.e1-4.e3

10. Gößwald $A$, Lange $M$, Kamtsiuris $P$, Kurth BM (2012) DEGS: Studie zur Gesundheit Erwachsener in Deutschland. Bundesweite Quer- und Längsschnittstudie im Rahmen des Gesundheitsmonitorings des Robert Koch-Instituts. Bundesgesundheitsbl Gesundheitsforsch Gesundheitsschutz 55:775-780

11. Scheidt-Nave C, Kamtsiuris P, Gößwald A et al (2012) German Health Interview and Examination Survey for Adults (DEGS) - design, objectives and implementation of the first data collection wave. BMC Public Health 12:730
12. Kamtsiuris $P$, Lange $M$, Hoffmann R et al (2013) Die erste Welle der Studie zur Gesundheit Erwachsener in Deutschland (DEGS1). Stichprobendesign, Response, Gewichtung und Repräsentativität. Bundesgesundheitsbl Gesundheitsforsch Gesundheitsschutz 56:620-630

13. Robert Koch-Institut (Hrsg) (2009) DEGS: Studie zur Gesundheit Erwachsener in Deutschland Projektbeschreibung. Beiträge zur Gesundheitsberichterstattung des Bundes. RKI, Berlin

14. Gößwald A, Lange M, Dölle R, Hölling H (2013) Die erste Welle der Studie zur Gesundheit Erwachsener in Deutschland (DEGS1). Gewinnung von Studienteilnehmenden, Durchführung der Feldarbeit und Qualitätsmanagement. Bundesgesundheitsbl Gesundheitsforsch Gesundheitsschutz 56:611-619

15. Mensink G, Burger M, Beitz R et al (2002) Was essen wir heute? Ernährungsverhalten in Deutschland. Robert Koch-Institut, Berlin

16. Truthmann J, Mensink GBM, Richter A (2011) Relative validation of the KiGGS Food Frequency Questionnaire among adolescents in Germany. Nutr J 10:133

17. Haftenberger M, Heuer T, Kube F et al (2010) Relative validity of a food frequency questionnaire for national health and nutrition monitoring. Nutr J 9:36

18. Lampert T, Kroll L, Müters S, Stolzenberg H (2013) Messung des sozioökonomischen Status in der Studie zur Gesundheit Erwachsener in Deutschland (DEGS1). Bundesgesundheitsbl Gesundheitsforsch Gesundheitsschutz 56:631-636

19. Heidemann C, Scheidt-Nave C, Richter A, Mensink GBM (2011) Dietary patterns are associated with cardiometabolic risk factors in a representative study population of German adults. $\mathrm{Br} J$ Nutr 106:1253-1262

20. Mróz LW, Chapman GE, Oliffe JL, Bottorff JL (2011) Men, food, and prostate cancer: gender influences on men's diets. Am J Mens Health 5:177187

21. Mensink GBM, Beitz R (2004) Food and nutrient intake in East and West Germany, eight years after the reunification - the German Nutrition Survey 1998. Eur J Clin Nutr 58:1000-1010

22. Heseker H, Gedrich K, Wagner K, Karg G (2008) Trendanalysen zum Lebensmittelverbrauch auf der Basis der Agrarstatistik. In: Ernährungsbericht 2008. Deutsche Gesellschaft für Ernährung e.V Bonn, S 20-36

23. Buchholz U, Bernard H, Werber D et al (2011) German outbreak of Escherichia coli 0104: H4 associated with sprouts. N Engl J Med 365:1763-1770

24. Robert Koch-Institut (2012) Daten und Fakten: Er-

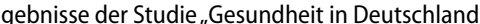
aktuell 2010". Beiträge zur Gesundheitsberichterstattung des Bundes. RKI, Berlin 